\title{
Predictors of Track Test Performance in Drivers with Stroke
}

\author{
Yasutaka KOBAYASHI ${ }^{1,3}$, Yutaro OMOKUTE ${ }^{2}$, Akiyo MITSUYAMA ${ }^{1}$, Yuka TAKAOKA², Chiaki TAKAMA ${ }^{1}$, \\ Yoko WATANABE ${ }^{2}$ \\ ${ }^{1}$ Fukui General Hospital, Department of Rehabilitation Medicine, Fukui, Japan \\ ${ }^{2}$ Fukui General Clinic, Department of Rehabilitation Medicine, Fukui, Japan \\ ${ }^{3}$ Fukui College of Health Sciences, Department of Rehabilitation Medicine, Fukui, Japan
}

\section{ABSTRACT}

AIM: To identify in-house assessment criteria to predict outcomes of driving tests in patients with stroke who wish to resume driving. MATERIAL and METHODS: A total of 181 patients with stroke who attended Fukui General Hospital (as outpatients or inpatients) between 2003 and 2015 and who had no obvious motor impairment were included in the study. All subjects underwent a neuropsychological examination, a driving simulator test, and a track test at a driving school. Based on their performance in the track test, the subjects were divided into capable drivers (CD group) and incapable drivers (ID group). Intergroup differences in test results were evaluated. Logistic regression analysis was performed using age and outcomes of Trail-Making Tests $A$ and $B$, and Symbol Digit Modalities Test (SDMT) as independent variables and track test performance as the dependent variable.

RESULTS: The ID group performed worse than the CD group in most aspects of the neuropsychological examination. There was no significant difference between the two groups with respect to any component of the driving simulator test. SDMT values were extracted from the logistic regression analysis, with an Odds Ratio of $1.05(\mathrm{p}=0.028)$. On receiver operating characteristic curve analysis (with the area under the curve of 0.76 ), at the SDMT achievement rate $<37.3 \%$, there was a sensitivity of $65 \%$ and specificity of $79 \%$ for the identification of driving inability.

CONCLUSION: Neuropsychological tests are useful for evaluating the ability of patients with stroke to resume driving. In particular, SDMT is the most suitable test for predicting driving test outcomes.

KEYWORDS: Automobile driving, Predictor, Stroke, Neuropsychological test, Driving test

\section{INTRODUCTION}

$\mathrm{D}$ riving is an invaluable skill in modern society, particularly in patients with a physical disability, in whom the ability to drive may serve as a key enabler and a determinant of their quality of life. In patients with disabilities, such as hemiplegia and paraplegia, ergonomic modifications in automobiles can greatly compensate for their disability while driving. However, the driving ability of patients with neuropsychological disturbance, such as those with attention deficit disorder, may be compromised regardless of the physical disability. In Japan, people with disability who have a driving license and who wish to resume driving are required to qualify an aptitude test (Table I) conducted by the Public Safety Commission (17). However, to draw judgments regarding the cognitive ability to drive safely necessitates medical evaluation. Overestimation of the driving ability of people with disabled is an obvious hazard to road safety, whereas any underestimation of their ability is liable to affect their social reintegration and quality of life. Therefore, judgments should be carefully drawn.

Evaluation of the ability of patients with brain injury to resume driving includes driving tests (track and road tests) conducted at driving schools and driving simulator and neuropsychological tests that are conducted at hospitals. 
Table I: Aptitude Test Criteria for Normal Driving Licenses

\begin{tabular}{|c|c|}
\hline Eyesight & $\begin{array}{l}\text { - } \quad \text { Bilateral visual acuity of } \geq 0.7 \text {, and } \geq 0.3 \text { in one eye. } \\
\text { In case of }<0.3 \text { vision in one eye, a vision of } \geq 0.7 \text { in the other eye, and a bilateral visual field of } \\
\geq 150^{\circ} \text {. }\end{array}$ \\
\hline Color vision & - Able to differentiate red, blue, and yellow \\
\hline Hearing & $\begin{array}{l}\text { - Capable of hearing the sound of a horn at } 90 \mathrm{~dB} \text { from } 10 \mathrm{~m} \text { away (or the ability to recognize an } \\
\text { automobile approaching from rear using the rearview mirror if incapable of the above hearing) }\end{array}$ \\
\hline Driving ability & $\begin{array}{l}\text { - Cognition and operation ability necessary for safe driving; } \\
\text { - Use of assistive means is permitted }\end{array}$ \\
\hline
\end{tabular}

Simplified excerpt from the Road Traffic Law Enforcement Regulations (revised 17th Dec, 2015).

Driving tests are considered to be the best measure of a person's driving ability and have been used as an outcome measure in several studies $(2,4,7,8,10,13,16,20,21)$. However, certain aspects of hazard prediction may not be amenable to assessment in a track test. Moreover, driving test performed on roads is an obvious road safety hazard. A driving simulator test can function as a substitute to driving tests; however, its ability to predict the driving ability of patients with stroke is unclear. Moreover, high cost and limited availability prevents their wider use. Neuropsychological tests are convenient to administer; however, predictions of driving ability differ among reports. Thus, although these evaluations have their own advantages and disadvantages, a more comprehensive evaluation is required to determine the driving ability of patients who are recovering from stroke to resume driving. However, conducting a comprehensive evaluation with all these tests causes a large financial and physical burden on patients and evaluators. Therefore, we attempted to predict the ability to resume driving in patients with stroke using a simple test.

\section{MATERIAL and METHODS}

\section{Patients}

A total of 181 patients with stroke (subarachnoid hemorrhage $(n=12)$, cerebral infarction $(n=118)$, and cerebral hemorrhage $(n=51)$, who were registered as inpatients or outpatients at the Fukui General Hospital between 2003 and 2015, were eligible for inclusion. The study was approved by the Ethical Review Board at the Nittazuka Medical Welfare Center.

Inclusion criteria were as follows: (1) patients who drove regularly for at least 10 years (prior to stroke) while having a valid driving license; (2) at least 3 months elapsed since onset of stroke; (3) the desire to resume driving; (4) ability to walk and maintain an upright sitting position and no physical impairment that would impair operation of the steering wheel, gas pedal, or brake pedal. Exclusion criteria were as follows: (1) an unstable general condition; (2) history of epileptic seizures in the preceding 2 years; (3) patients with dementia; (4) mental illness such as depression; (5) failure to meet any of the criteria set by the Public Safety Commission regarding eyesight, field of vision, and hearing (17). Written informed consent was obtained from all patients before their enrolment in the study. All subjects underwent neuropsychological examination and a driving simulator at the hospital and a track test at a driving school.

\section{Neuropsychological Examination}

All patients underwent the Trail Making Test A (TMT-A), Trail Making Test B (TMT-B), Mini Mental State Examination (MMSE), Digit Span (DS), Tapping Span (TS), Visual Cancellation Task (VCT), Auditory Detection Task (ADT), Symbol Digit Modalities Test (SDMT), Memory Updating Test (MUT), Paced Auditory Serial Addition Test (PASAT), and Position Stroop Test (PST).

\section{Driving Simulator}

Patients recruited from year 2012 onwards underwent a driving simulation test using a Honda Safety Navi system (Honda Motor Co., Tokyo, Japan). Simple reaction, selective reaction, divided attention multitasking, and steering wheel operation were evaluated. The simple reaction test involved virtual driving of the car on the screen using the gas pedal, while following visual cues from monochromatic lights that lit up at fixed positions on the screen. The selective reaction test involved use of gas and brake pedals, while following visual cues (three different colored lights) that lit up at fixed positions on the screen. In the divided attention multitasking test, drivers had to follow visual cues from three different colored lights that lit up at unknown positions on the screen and by manually pressing buttons according to the arrow signs on the screen. Steering wheel operation involved virtually steering the car between traffic cones. All tests were capable of testing reaction speed and accuracy.

\section{Driving Test (Track Test)}

The driving test was conducted at an affiliated driving school. Before evaluation, patients responded to an interview associated with their health and underwent blood pressure evaluation. In case of any obvious abnormalities, the patients' eligibility to undergo evaluation was determined after consultation with their attending physician.

In general, the instructor from the driving school sat in the front passenger seat and an occupational therapist or family member sat in one of the rear seats. The patients drove the vehicle at the driving course for approximately 50 minutes. Sixteen aspects were evaluated (Table II), and the patients 
had to drive on the same course twice. Items were marked "possible" if the patient was deemed capable of achieving them while driving alone or with a family member. Finally, if all items were deemed "possible" by the second lap of the course, the patient was judged to be "capable of driving;" however, if that was not the case, the patient was judged to be "incapable of driving."

\section{Statistical Analysis}

A chi-square test was used to compare disease and sex between drivers who were deemed to be capable of driving (CD group) and those who were incapable of driving (ID group), whereas an independent $t$-test was used to compare age. Results of the neuropsychological examination and the driving simulator test between the two groups were compared using the Mann-Whitney $U$ test.

A stepwise logistic regression analysis was conducted with age, TMT-A, TMT-B, and SDMT as independent variables and driving capability as the dependent variable. The independent variables were selected with reference to past literature (13, 18,19). In addition, considering the effect of multicollinearity, a correlation matrix was established before analysis, and the variance inflation factor (VIF) was calculated. Independent variables that were extracted using logistic regression analysis were used to obtain a receiver operating characteristic (ROC) curve, which was used to identify the optimal cutoff value.

All statistical analyses were performed using BellCurve for Excel software (Social Survey Research Information Co., Ltd., Tokyo, Japan). Significance was set at a level of $5 \%$.

\section{RESULTS}

\section{Driving Test (Track Test)}

Based on the driving test, 128 (105 men, 23 women; Mean age, $56.0 \pm 10.6$ years) subjects were deemed capable of driving (CD group) and 53 (48 men and five women; Mean age, 62.8 \pm 8.9 years) deemed incapable of driving (ID group).

\section{Characteristics of Patients}

Diseases in the CD group were subarachnoid hemorrhage $(n=9)$, cerebral infarction $(n=82)$, and cerebral hemorrhage $(n=37)$ subjects, whereas those in the ID group were subarachnoid hemorrhage $(n=3)$, cerebral infarction $(n=36)$ and cerebral hemorrhage $(n=14)$. No significant intergroup differences were observed with respect to mean age, sex distribution, and diseases (Table III).

\section{Neuropsychological Examination}

The Mann-Whitney $U$ test revealed significantly poorer results in the ID group than in the CD group, with respect to the following variables: TMT-A (time required), TMT-B (time required), MMSE (total score), DS-forward (maximum number of digits), DS-backward (maximum number of digits), TSforward (maximum number of digits), TS-backward (maximum number of digits), VCT-shape 1 (time required and accuracy rate), VCT-shape 2 (time required), VCT-number "3" (time required), VCT-letter "ka" (time required and accuracy rate), ADT (accuracy rate and hitting rate), SDMT (achievement rate), MUT-3 digits (accuracy rate), MUT-4 digits (accuracy rate), PASAT-2 seconds (accuracy rate), and PASAT-1 second (accuracy rate) (Table IV).

Table II: Evaluation Items in the Track Test

\begin{tabular}{ll}
\hline 1. Brake pedal operation & 9. Reaction to obstacles \\
\hline 2. Handling of curves and turns & 10. Following lights, signs and markings \\
\hline 3. Lane choice & 11. Navigating intersections with poor visibility \\
\hline 4. Route changes & 12. Reversing \\
\hline 5. Navigating narrow roads & 13. Taking in information \\
\hline 6. Navigating intersections (straight on) & 14. Memory skills \\
\hline 7. Navigating intersections (turning left) & 15. Situational judgment \\
\hline 8. Navigating intersections (turning right) & 16. Overall content \\
\hline
\end{tabular}

Table III: Characteristics of Patients According to the Outcome of the Driving Test

\begin{tabular}{|c|c|c|c|}
\hline & Capable drivers group $(n=128)$ & Incapable drivers group $(n=53)$ & \\
\hline Disease (SAH/Cl/ICH) & $9 / 82 / 37$ & $3 / 36 / 14$ & n.s. \\
\hline Sex (Male/Female) & $105 / 23$ & $48 / 5$ & n.s \\
\hline Age (mean $\pm S D)$ & $56.0 \pm 10.6$ & $62.8 \pm 8.9$ & n.s \\
\hline
\end{tabular}

SAH: Subarachnoid hemorrhage, CI: cerebral infarction; ICH: Intracerebral hemorrhage; n.s.: Not significant; SD: standard deviation. 
Table IV: Neuropsychological Examination

\begin{tabular}{|c|c|c|c|c|c|}
\hline & & & $\begin{array}{l}\text { Capable drivers group } \\
\qquad(n=128)\end{array}$ & $\begin{array}{l}\text { Incapable drivers group } \\
\qquad(\mathrm{n}=53)\end{array}$ & \\
\hline TMT-A & & Time required (seconds) & $40.5(33.0-54.0)$ & $54.5(46-68.3)$ & $\mathrm{p}<0.01$ \\
\hline TMT-B & & Time required (seconds) & $91.0(65.0-122.0)$ & $123.5(98.0-178.0)$ & $p<0.01$ \\
\hline MMSE & & total score (point) & $29(27-30)$ & $27(24-28)$ & $p<0.01$ \\
\hline \multirow[t]{2}{*}{ DS } & forward & maximum number of digits & $6(5-6.5)$ & $5(4-6)$ & $p<0.01$ \\
\hline & backward & maximum number of digits & $5(4-5)$ & $4(3-5)$ & $p<0.01$ \\
\hline \multirow[t]{2}{*}{ TS } & forward & maximum number of digits & $6(5-7)$ & $5(4-6)$ & $\mathrm{p}<0.01$ \\
\hline & backward & maximum number of digits & $5(4-6)$ & $5(3-6)$ & $p<0.01$ \\
\hline \multirow[t]{8}{*}{ VCT } & shape1 & Time required (seconds) & $50.0(43.5-58.5)$ & $58.0(52.0-71.0)$ & $\mathrm{p}<0.01$ \\
\hline & & accuracy rate (\%) & $100(98-100)$ & $98.2(94.7-100)$ & $\mathrm{p}<0.05$ \\
\hline & shape2 & Time required (seconds) & $58.0(51.0-74.0)$ & $72.0(63.0-92.0)$ & $p<0.01$ \\
\hline & & accuracy rate (\%) & $100(98.2-100)$ & $100(98-100)$ & n.s. \\
\hline & number & Time required (seconds) & $98.0(85.5-117.5)$ & $110.0(94.0-138.0)$ & $p<0.01$ \\
\hline & & accuracy rate (\%) & $99.1(98.1-100)$ & $99.1(98-100)$ & n.s. \\
\hline & letter & Time required (seconds) & $124.0(105.5-150.5)$ & $145.0(116.0-174.0)$ & $\mathrm{p}<0.05$ \\
\hline & & accuracy rate (\%) & $97.4(93.9-99.1)$ & $94.7(88-98)$ & $p<0.01$ \\
\hline \multirow[t]{2}{*}{ ADT } & & accuracy rate (\%) & $96(94-98)$ & $90(78-96)$ & $\mathrm{p}<0.01$ \\
\hline & & hitting rate (\%) & $92.5(66.6-98)$ & 73.6 (41.3-92) & $p<0.01$ \\
\hline SDMT & & achievement rate (\%) & $41.8(31.4-47.7)$ & $30(22.7-36.4)$ & $p<0.01$ \\
\hline \multirow[t]{2}{*}{ MUT } & 3 digits & accuracy rate (\%) & $75(56.3-90.3)$ & $56.3(31.3-81)$ & $p<0.01$ \\
\hline & 4 digits & accuracy rate (\%) & $56.3(37.5-68.8)$ & $37.5(0-50)$ & $\mathrm{p}<0.01$ \\
\hline \multirow[t]{2}{*}{ PASAT } & 2 seconds & accuracy rate (\%) & $50(33.3-65)$ & $36.7(18.3-45)$ & $p<0.01$ \\
\hline & 1 second & accuracy rate (\%) & $26.7(20-39.2)$ & $18.3(0-26.7)$ & $p<0.01$ \\
\hline PST & & accuracy rate (\%) & $98.2(96.5-100)$ & $97.4(93.9-99.1)$ & n.s. \\
\hline
\end{tabular}

TMT-A: Trail Making Test A, TMT-B: Trail Making Test B, MMSE: Mini Mental State Examination, DS: Digit Span, TS: Tapping Span, VCT: Visual Cancellation Task, ADT: Auditory Detection Task, SDMT: Symbol Digit Modalities Test, MUT: Memory Updating Test, PASAT: Paced Auditory Serial Addition Test, PST: Position Stroop Test, n.s.: not significant.

\section{Driving Simulator}

No significant intergroup difference in performance was observed in simple reaction, selective reaction, and divided attention multitasking tests and steering wheel operation (Table V).

\section{Logistic Regression}

VIF was $<10$ in all independent variables, indicating no issues associated with multicollinearity. Stepwise logistic regression analysis with age, TMT-A, TMT-B, and SDMT outcomes as independent variables, and the track test performance as the dependent variable, identified a significant correlation between SDMT outcomes (achievement rate) and track test results (Odds Ratio [OR]: 1.05 [ $p=0.028]$; Table VI). On ROC curve analysis (AUC 0.76), at the SDMT achievement rate of $<37.3 \%$, the sensitivity was $65 \%$ and specificity was $79 \%$ for identification of driving inability (Figure 1).

\section{DISCUSSION}

Driving is a complex task that requires attention, memory, judgment, and other cognitive functions in addition to vision, hearing, and physical function. Michon (14) categorized driving into a strategic level, a tactical level, and an operational level. The strategic level requires planning (destination, route, and cost); the operational level requires basic maneuvering capability (steering and braking). Any deficits at these levels can be compensated for by the presence of a passenger or modification of the car. However, abilities associated 
Table V: Driving Simulator

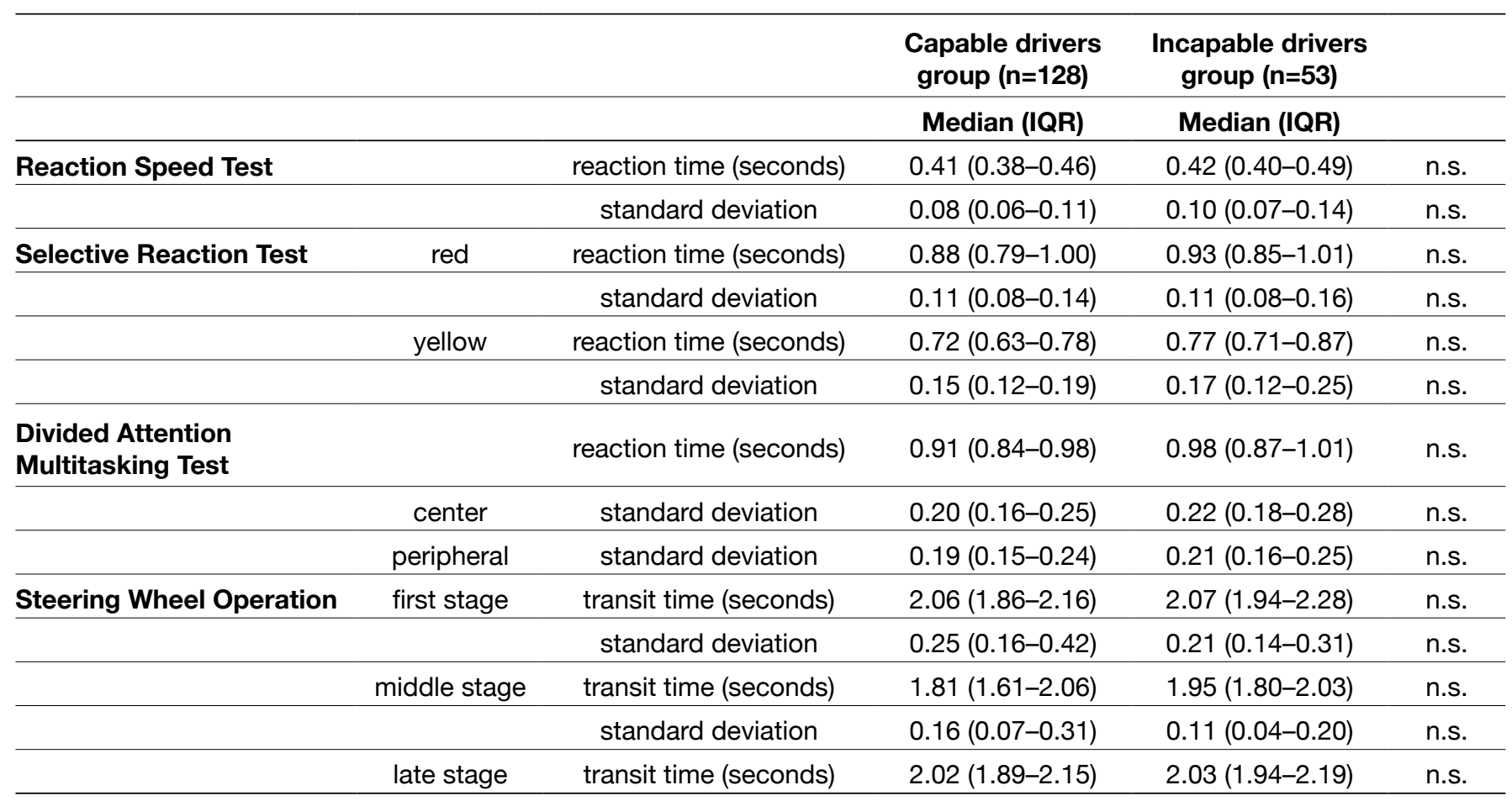

IQR: interquartile range; n.s.: not significant.

Table VI: Logistic Regression Analysis

\begin{tabular}{|c|c|c|c|}
\hline & Odds Ratio & $95 \% \mathrm{Cl}$ & p Value \\
\hline Age & 0.968 & 0.928 to 1.010 & 0.132 \\
\hline TMT-A & 0.989 & 0.964 to 1.015 & 0.392 \\
\hline TMT-B & 0.996 & 1.006 to 1.104 & 0.291 \\
\hline SDMT (achievement rate) & 1.054 & 1.104 to 4.837 & 0.028 \\
\hline
\end{tabular}

TMT: Trail Making Test, SDMT: Symbol Digit Modalities Test, Cl: Confidence interval.

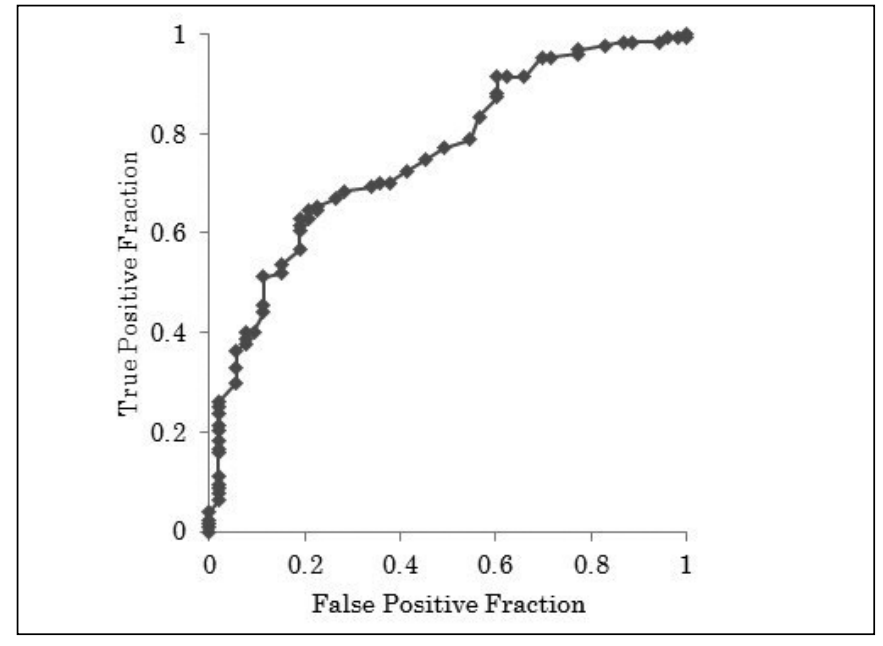

Figure 1: Receiver Operating Characteristic curve. The area under the curve was 0.76 , and at an SDMT achievement rate $<37.3 \%$, there was a sensitivity of $65 \%$ and a specificity of $79 \%$ for identification of driving inability. with the tactical level such as speeding up, slowing down, turning off, or overtaking are strongly associated with mental faculties (attention, memory, and judgment) and cannot be compensated for using external aids. Therefore, judgment of these abilities requires meticulous assessment. Here, we evaluated the different aspects of assessment of driving ability at the tactical level.

Univariate analysis revealed poorer performance of subjects in the ID group in all neuropsychological tests except VCT (shape 2, number) accuracy rate, and PST accuracy rate than those in the CD group. An earlier review of 17 studies that examined predictors of driving ability post-stroke found that cognitive ability was the most useful screening test (12). We used multivariate analysis to identify predictors of track test performance from the results of neuropsychological tests. Studies have examined predictors for road test performance from the outcomes of TMT-A (13), TMT-B (13), Rey-Osterreith Complex Figure test (ROCF) (1), SDMT $(18,19)$, and Useful Field of View test (UFOV) $(1,5)$. Based on the results of these 
studies, we included outcomes of TMT-A, TMT-B, SDMT, and age as independent variables, and track test performance as the dependent variable in the logistic regression model. SDMT was significantly correlated with track test performance. ROC curve for SDMT had an AUC of 0.76 which indicated a significant predictive ability. Furthermore, at an SDMT achievement rate of $37.3 \%$ as reference, use of SDMT as a predictor of driving ability was associated with a sensitivity and specificity of $65 \%$ and $79 \%$, respectively. Driving involves the ability to direct one's attention toward multiple objects in the field of vision, to switch one's object of attention depending on the circumstances, and to control driving speed among other abilities. However, these require the use of mental faculties at a tactical level, as demonstrated by Michon (14). These abilities are equivalent to the division, switching, and control of attention associated with SDMT extracted in the present study.

The driving simulation test was performed in patients recruited from 2012 onwards when the simulator was introduced. Few reports have examined the driving abilities of patients with stroke using a driving simulator. Studies have shown that patients with stroke tend to perform worse on driving simulators than healthy individuals; however, this association was not observed with regard to road driving $(9,11)$. The driving simulator used in the present study enables the evaluation of the course of travel and reactions and steering wheel operation. However, the course of travel was excluded from the present study items as the objective was to predict track driving at a driving school. Therefore, no significant intergroup differences were observed with respect to results of the driving simulator test. Moreover, all subjects did not perform the driving simulator test; therefore, this test was not used as an independent variable in the logistic regression analysis. The advantage of driving simulators is that they can predict risk factors that driving test evaluations cannot sufficiently predict; therefore, further study with regard to simulator tests is required.

In this study, $70 \%$ of the subjects successfully qualified the track test. According to a review of 16 earlier studies (6), the corresponding percentage of patients who were capable of driving ranged widely between $23.68 \%$ and $83.72 \%$, and only three reports cited rates $>70 \%$; the rate in our study was high. This is possibly because of the effect of different test methods, envisaged traffic conditions, and test judgment criteria. Because the present study included a track test, evaluation on the assumption of driving in a provincial city with relatively less traffic than that in a large city, and a judgment of "capable" when a family member was a passenger, the percentage of patients found capable of driving was higher.

A road test $(2,4,7,8,10,13,20,21)$, track test (16), driving status (return to driving post-stroke) (15), questionnaire (3), driving simulator (9) and other tests were used to set outcomes. A road test is generally considered to be the gold standard for evaluation of driving ability (12); however, several facilities in Japan use a track test. Regardless of the setting, evaluation of driving ability requires resources for both patient and the driving school. To prevent this, attempts have been performed to identify potential screening tests for predicting the outcome of actual driving tests in vehicles. The capability of screening tests to predict driving ability is likely to save resources, and to provide information on driving ability to patients and the Public Safety Commission. The results of the present study indicate a strong likelihood of failing a track test because the SDMT achievement rate does not exceed $37.3 \%$. In other words, SDMT test outcome can be used as a decision tool, e.g., to recommend the use of public transport.

Some limitations of the study should be considered for interpreting our results. The use of track test performance as an outcome measure is one of the limitations. A road test is considered a valid and reliable test for evaluating driving ability post-stroke and has been used in several studies as the gold standard (6). However, a road test is associated with the risk of traffic accidents during evaluation, which makes the locus of responsibility a potential issue. Second, we did not include ROCF or UFOV tests, which have been reported as predictors of driving ability in several studies. The UFOV test is not widely available in Japan, and further studies are required. Third, patients with clear motor impairment were excluded from the study. In actual clinical settings, several patients with coexisting motor impairment and sensory dysfunction exist. A future study is required to examine the effect of various types of disabilities on the validity of our findings. Accordingly, a multicenter study with a larger sample size may provide robust evidence.

\section{CONCLUSION}

Neuropsychological tests are useful as screening tests for evaluating the ability of patients with stroke to resume driving. These tests may eliminate or minimize the requirement for actual driving tests. SDMT appears to be a potential predictor of track test performance.

\section{- REFERENCES}

1. Akinwuntan $A E$, Feys $H$, DeWeerdt W, Pauwels J, Baten G, Strypstein E: Determinants of driving after stroke. Arch Phys Med Rehabil 83: 334-341, 2002

2. Akinwuntan AE, DeWeerdt $W$, Feys $H$, Baten G, Arno P, Kiekens C: The validity of a road test after stroke. Arch Phys Med Rehabil 86: 421-426, 2005

3. Fisk GD, Owsley C, Mennemeier M: Vision, attention, and self-reported driving behaviors in community-dwelling stroke survivors. Arch Phys Med Rehabil 83: 469-477, 2002

4. George S, Clark M, Crotty M: Validation of the Visual Recognition Slide Test with stroke: A component of the New South Wales occupational therapy off-road driver rehabilitation program. Aust Occup Ther J 55: 172-179, 2008

5. George S, Crotty M: Establishing criterion validity of the useful field of view assessment and stroke drivers' screening assessment: Comparison to the result of on-road assessment. Am J Occup Ther 64:114-122, 2010

6. Hird MA, Vetivelu A, Saposnik G, Schweizer TA: Cognitive, onroad, and simulator-based driving assessment after stroke. J Stroke Cerebrovasc Dis 23:2654-2670, 2014 
7. Klavora P, Heslegrave RJ, Young M: Driving skills in elderly persons with stroke: Comparison of two new assessment options. Arch Phys Med Rehabil 81:701-705, 2000

8. Korner-Bitensky NA, Mazer BL, Sofer S, Gelina I, Meyer MB, Morrison C, Tritch L, Roelke MA, White M: Visual testing for readiness to drive after stroke: A multicenter study. Am J Phys Med Rehabil 79: 253-259, 2000

9. Kotterba S, Widdig W, Brylak S, Orth M: Driving after cerebral ischemia-a driving simulator investigation. Wien Med Wochenschr 155: 348-353, 2005

10. Lundberg C, Caneman G, Samuelsson SM, HakamiesBlomqvist L, Almkvist O: The assessment of fitness to drive after a stroke: The Nordic Stroke Driver Screening Assessment. Scand J Psychol 44: 23-30, 2003

11. Lundqvist A, Gerdle B, Ronnberg J: Neuropsychological aspects of driving after stroke-in the simulator and on the road. Appl Cognitive Psycho 14: 135-150, 2000

12. Marshall SC, Molnar F, Man-Son-Hing M, Blair R, Brosseau L, Finestone HM, Lamothe C, Korner-Bitensky N, Wilson KG: Predictors of driving ability following stroke: A systematic review. Top Stroke Rehabil 14: 98-114, 2007

13. Mazer BL, Korner-Bitensky NA, Sofer S: Predicting ability to drive after stroke. Arch Phys Med Rehabil 79: 743-750, 1998

14. Michon JA: Dealing with danger. Summary report of a workshop on physiological and psychological factors in performance under hazardous conditions with special reference to road traffic accidents. Gieten: Medical Research Committee of the Commission of the European Communities, 1978: 1-20
15. Perrier MJ, Korner-Bitensky N, Mayo NE: Patient factors associated with return to driving post-stroke: Findings from a multicenter cohort study. Arch Phys Med Rehabil 91: 868-873, 2010

16. Ponsford AS, Viitanen M, Lundberg C, Johansson K: Assessment of driving after stroke-a pluridisciplinary task. Accid Anal Prev 40: 452-460, 2008

17. Public Safety Commission, Road Traffic Law Enforcement Regulations. December 17, 2015. Available at: http://law.egov.go.jp/htmldata/S35/S35F03101000060.html.

18. Rapport LJ, Bryer RC, Hanks RA: Driving and community integration after traumatic brain injury. Arch Phys Med Rehabil 89: 922-930, 2008

19. Schanke AK, Sundet K: Comprehensive driving assessment: Neuropsychological testing and on-road evaluation of brain injured patients. Scand J Psychol 41: 113-121, 2000

20. Selander H, Johansson K, Lundberg C, Falkmer T: The Nordic stroke driver screening assessment as predictor for the outcome of an on-road test. Scand J Occup Ther 17:10-17, 2010

21. Söderström ST, Pettersson RP, Leppert J: Prediction of driving ability after stroke and the effect of behind-the-wheel training. Scand J Psychol 47: 419-429, 2006 